\title{
VIVIR A LOS TUMBOS O VIVIR (DE) UNIFORME: BIOGRAFÍAS DE JÓVENES POLICÍAS EN ARGENTINA
}

\author{
TOMÁS BOVER* \\ MARIANA CHAVES ${ }^{* *}$
}

\begin{abstract}
RESUMEN
El artículo se centra en el análisis de relatos biográficos de personas jóvenes que ingresan en fuerzas de seguridad estatales con el objetivo de analizar la composición de las trayectorias sociales e interpretar cómo se articulan las constricciones de las condiciones de vida con la capacidad de agencia de los sujetos en la toma de decisiones sobre sus opciones de estudio, trabajo y proyectos de vida. A partir de esto, se desarrollan en el escrito discusiones en torno a: i) la linealidad de las trayectorias con trabajo de tipo pleno empleo; ii) la asociación entre inseguridad y empleo que se produce en el trabajo de policía; iii) las formas que cobra la experiencia juvenil como trabajadores de fuerzas de seguridad, y finalmente, iv) las transformaciones que se producen o no en las relaciones familiares, de amistad y en la propia persona a partir de formarse como policía.
\end{abstract}

PALABRAS CLAVE: TRAYECTORIA, POLICÍA, JÓVENES

* Antropólogo, doctorando en IDAES (UNSAM), becario Núcleo de Estudios Socioculturales, Facultad de Trabajo Social, Universidad Nacional de La Plata. Correo electrónico: tomasbover@yahoo.com.ar.

** Antropóloga, profesora de la Universidad Nacional de La Plata (UNLP) y Universidad Tres de Febrero (UNTREF). Investigadora Núcleo de Estudios Socioculturales, Facultad de Trabajo Social, Universidad Nacional de La Plata/CONICET. Correo electrónico:mchaves@fcnym.unlp.edu.ar.

Una versión previa de este artículo fue presentada y discutida en «XVII ISA World Congress of Sociology Sociology on the move», Gotemburgo, Suecia, 11 al 17 de julio de 2010. 


\title{
VIVER AO LÉU OU VIVER (D UNIFORME: BIOGRAFIAS DE JOVENS POLICIAIS NA ARGENTINA
}

\begin{abstract}
RESUMO
O foco do artigo é a análise de relatos biográficos de pessoas jovens que ingressam em forças estatais de segurança com o objetivo de analisar elementos das trajetórias sociais e interpretar como se articulam as tensões das condições de vida com a capacidade de agência dos sujeitos na tomada de decisões sobre suas opções de estudo, trabalho e projetos de vida. Nesse sentido, são desenvolvidas discussões sobre os seguintes temas: i) a linearidade das trajetórias com trabalho do tipo pleno emprego; ii) a associação entre insegurança e emprego que se produz no trabalho da polícia; iii) as formas que adquire a experiência juvenil como trabalhadores de forças de segurança, e finalmente iv) as transformações que se produzem ou não nas relações familiares, de amizade e na própria pessoa a partir da formação como policial.
\end{abstract}

PALAVRAS CHAVE: TRAJETÓRIA, POLÍCIA, JOVENS

\section{TO LIVE WITH DIFFICULTY OR TO LIVE IN UNIFORM: BIOGRAPHIES OF YOUNG POLICEMEN IN ARGENTINA}

\begin{abstract}
This article is centered on the analysis of biographies related to young people who apply to work in state security forces with the objective to analyze the composition of social paths in order to interpret how these constricted conditions of life affect the lives of its people on making decisions regarding their options of study, work, and other life projections. To start with, a development in written discussions revolve around: i) the line of complete job type, ii) the association between insecurity and employment which is produced through the police service, iii) the ways in which the youth experience work in the security forces is formed, and finally, iv) the transformation that it does or does not produce en regards to family relationships, friendship and self formation in the police service.
\end{abstract}

KEY WORDS: PATH, POLICY, YOUTH 


\section{INTRODUCCIÓN: LAS CIRCUNSTANCIAS}

ESTE TRABAJO SE CENTRA en el análisis de relatos biográficos de personas jóvenes que trabajan o estudian en la policía de la Provincia de Buenos Aires, Argentina. Para ser policía, el primer paso institucional es inscribirse y entrar a estudiar becado ${ }^{1}$ en la escuela de formación durante algunos meses, para egresar generalmente al año ${ }^{2}$ con el título de Técnico Superior en Seguridad Pública y el cargo de Oficial de Policía del Agrupamiento Comando de la Policía de la Provincia de Buenos Aires, que involucra un puesto de trabajo estatal con derechos y responsabilidades que le competen a él/ella y su grupo familiar (salario, vacaciones, cobertura en salud, jubilación, seguro de vida, entre otros).

En la página del Ministerio de Justicia y Seguridad, se indican los requisitos de ingreso «tener entre 18 y 30 años; poseer condiciones intachables de moralidad y buenas costumbres; contar con el ciclo polimodal o secundario completo, sin adeudar materias; responder a las aptitudes psicofísicas que requiera el Ministerio de Seguridad. Masculinos: Tener una estatura no inferior a 1,60 metros y femeninos: tener una estatura no inferior a 1,56 metros» (www.mseg.gba.gov.ar). No hay registro de condición socioeconómica de los ingresantes, pero es plausible inferir a partir de las investigaciones realizadas en el tema que las policías se nutren en Argentina de jóvenes de clases bajas y medias con escolarización secundaria completa.

Las policías ${ }^{3}$ en nuestro país, como en todas partes del mundo, son una de las fuerzas para la aplicación legítima de la violencia por parte del Estado. Su ejercicio en un contexto de gobierno democrático ha sido muy distinto que en gobiernos dictatoriales. Durante los gobiernos militares - $\mathrm{y}$ en los años previos al golpe militar de 1976, desde 1974 - los miembros de las fuerzas policiales primero, y luego de la institución en pleno, estuvieron bajo el mando de las fuerzas armadas y actuaron en secuestros, desapariciones, torturas, encierros, apropiaciones, robos y asesinatos perpetrados en ese período. El saldo

1 Perciben un estipendio de manutención.

2 En algunas oportunidades, por necesidad de personal policial o transformaciones en la institución, la formación policial duró 6 meses.

3 El poder de policía se organiza por jurisdicciones, existiendo una policía dependiente de cada estado provincial y otras de jurisdicción federal. 
en vidas humanas cuenta por miles. Una de sus consecuencias simbólicas es el refuerzo de la idea que ser policía es ser alguien que infringe la ley, que no la cumple.

Pero el vínculo conflictivo de policía y juventud no aparece en ese período, es muy anterior. Su intervención se remonta a los inicios históricos de la institución, ya que una de sus primeras funciones fue y sigue siendo el control del orden en el espacio público, y éste ha sido también desde siempre el lugar elegido por los jóvenes para estar. Muchas veces la sola presencia juvenil —que se leía como indeseable por algunos sectores - habilitaba la intervención. En otras oportunidades, las disputas entre grupos de jóvenes era el llamado que permitía el ingreso del uso de la fuerza.

Los jóvenes siempre han sido mirados y descritos dualmente. Por un lado, la belleza, lozanía y diversión del modelo de joven en moratoria social, con la responsabilidad del estudio y la diversión los fines de semana, aprovechando el tiempo libre, consumiendo y desarrollando una sociabilidad despreocupada. Un modelo hegemónico de representación de la experiencia juvenil construido sobre las prácticas de los sectores altos y medios altos. Y por otra parte, los delincuentes, pibes chorros, jóvenes peligrosos, chicos de los barrios segregados, estigmatizando homogéneamente a todos/as los jóvenes pobres. La policía siempre estuvo junto a ellos para golpearlos, para apresarlos, también para corromperlos, y algunas veces, en contadas ocasiones, para cuidarlos.

\section{RELACIONES: FAMILIA, AMIGOS Y PAREJAS}

Las familias tienen modos particulares y diversos de enfrentar el hecho de que alguno/s de sus miembros ingresen a la policía. Esa circunstancia de la vida familiar se aborda de diferente manera, dependiendo de la experiencia que cada uno haya tenido con la institución y con sus agentes, y según la proyección de futuro que se había soñado para el familiar que decide ingresar. Las relaciones con los padres, madres, hermanos/as, amigos y parejas se modifican. Por una parte, está el impacto sobre la organización familiar y en las redes de amistad, debido a la ausencia del aspirante durante la semana y de su presencia sólo de fin de semana. Por otro lado, porque se registran cambios en el carácter y en las concepciones de los aspirantes que llevan a revisar y rearmar las relaciones en sus grupos de pertenencia. 


\section{a) Entre la familia y la policía}

Algunas de las actitudes de los padres que valoran los jóvenes dice relación con la contención o aguante que se manifiesta con la nueva situación. Se espera que los padres sean capaces de sostener a sus hijos afectiva y económicamente; por ejemplo, en el cuidado de sus nietos durante el período de formación. También que una vez ingresado en la fuerza policial, se espera que la familia acompañe y comprenda el ritmo extenso y cambiante de trabajo y los distintos estados de ánimo al volver a casa. Estos cambios se manifiestan en el humor, o carácter —ahora forjado como veremos más adelante-; la falta de predisposición para atender labores cotidianas que antes estaban a su cargo (por ejemplo, en el caso de las mujeres atender a padres y hermanos hombres al interior de su hogar); las nuevas elecciones del uso del tiempo libre; asumir un tono riguroso y una forma de comunicación imperativa aprendida o experimentada en la escuela o el trabajo, que ahora se traslada al vínculo al interior del hogar, y que antes no era acostumbrada.

Necesitamos realizar aquí una consideración. Los/as jóvenes aspirantes comparten una característica fundamental entre sí y con muchos de sus compañeros de trabajo de otras edades: su origen de clase. La mayoría proviene de sectores de clase que históricamente acceden a puestos de trabajo temporarios y cuando lo logran, generalmente son formas de trabajo no registrado (sin cobertura de salud ni sindicalización) y con bajos salarios. Este origen de clase produce una lectura familiar de la nueva posición de trabajo como un ascenso de clase, como una mejora en la trayectoria social del grupo, encarnada en la biografía del miembro de la familia que ha logrado ingresar al mercado de trabajo formal con un trabajo registrado: oficial de policía.

Esta percepción positiva entra en conflicto con la caracterización negativa que los/as policías tiene sobre algunos aspectos de las condiciones de su trabajo y que pareciera desconocen o subestiman los familiares. Sensaciones como el riesgo, miedo, aburrimiento, soledad, frío, calor, carga y la violencia que marcan su labor son huellas que trazan malestar en su vida laboral e impiden, o son insuficientes, para manifestar una satisfacción plena con el trabajo.

El conflicto por la administración del tiempo entre la familia y la policía tiene un nuevo capítulo cuando la persona ya no vive en el hogar de los padres y arma su propia familia en otra vivienda. Este 
punto ha sido también descrito por Sabina Frederic (2008, 2008b) en su investigación sobre la misma fuerza al analizar los usos y nociones del tiempo por parte de los policías. La autora resalta la dificultad que enuncian los agentes para gerenciar su tiempo libre y dedicárselo, entre otras cosas, a sus familias, tornándose problemático sostener una pareja estable y un vínculo de presencia con los hijos.

Esta situación es provocada por distintos motivos que muchas veces se superponen y potencian la ausencia de tiempo fuera del tiempo de servicio. Un caso puede ser la extensa jornada laboral. Otro, los traslados a dependencias alejadas del lugar de residencia que pueden tener como consecuencia la mudanza, dejando el núcleo familiar por largos períodos de tiempo; por ejemplo, alquilando otra vivienda, viviendo en alojamientos provistos por la propia policía, o pasar algunos días seguidos en la dependencia de trabajo. En estos últimos casos aparece una profundización de la sensación de ausencia de tiempo para estar con otros no policías ya que al compartir la vivienda y las horas no laborales con miembros de la fuerza, los temas de conversación redundan sobre la tarea policial, los hechos, o las anécdotas institucionales. Una entrevistada lo expresaba así:

Policía: ....ahí estás rodeada todo el día de policías, me quedaba a dormir en Avellaneda [una ciudad que dista unos $40 \mathrm{~km}$ de la ciudad donde ella vive] que había dormitorio, para no ir y venir de Avellaneda me quedaba a dormir allá y... estaba al lado de cinco policías más, me iba a bañar y habían cinco policías bañándose, eh... salía y estaba tooodo el día con policías, todas mujeres, y:

— ¿Viste lo que me pasó el otro día en el servicio?

- Ah que copado?

— ¿Viste lo que me pasó en el móvil? ¿Viste?

Y todo el día hablar de eso!...

\section{b) Amigos}

Ser policía no es una condición, más ni una característica con el mismo peso que ser carpintero o chofer al interior de los grupos de pares. Los amigos tienen que encontrar cómo resolver lo que aparece como una contradicción: que un miembro de su grupo sea parte de una institución que históricamente mantiene un vínculo represivo con ellos. La solución puede tomar distintas formas.

La investigación de Mariana Sirimarco $(2004,2009)$ con policías en formación sostiene que la sociabilidad en los grupos de pares varía 
con el ingreso a la policía, estructurando una narración de las relaciones entre pares que oponen un antes, con típica sociabilidad de grupo de esquina, y un ahora policía, donde esas prácticas ya no tienen cabida. La autora muestra, a través del relato de un cadete, cómo el joven refuerza su pertenencia actual a la fuerza, contrastándola con su antigua adscripción al grupo de pares barrial, presentando como incompatibles ambas adscripciones y estructurando un relato de sociabilidad juvenil dividido entre un antes y un después de hacerse policía.

En nuestra investigación hemos encontrado también esta forma de procesar la contradicción, construyendo un quiebre en la narración biográfica para mantener separada la sociabilidad juvenil de civiles y policías. Pero también hemos hallado otra solución para esta circunstancia.

La condición de policía será ineludible, no es entonces posible esconderla, ocultarla a aquéllos con los que se viene compartiendo afecto y diversión. No se trata entonces de dejar los amigos ni dejar de ser policía, sino de procesar ambas pertenencias. Esto encuentra una resolución en la risa. Los jóvenes harán chistes sobre ser parte del grupo y pertenecer a una fuerza de seguridad; a través de jodas, bromas, sobrenombres o gastadas se procesará la supuesta contradicción. Reconocimos tres formas de esta resolución:

i) Las bromas que toman como objeto de burla al amigo/a policía permiten tomarse revancha de la institución policial que tantas veces los ha enfrentado. Son revanchas personales por experiencias de enfrentamientos directos y también revanchas sectoriales, al tomar la voz como referentes de los jóvenes o los reprimidos por la policía. Reírse de la policía es factible, porque el amigo/a no va a reaccionar en el grupo de pares del mismo modo que otros policías lo harían, o hasta él mismo en otros contextos.

ii) Bromas que toman como eje la posibilidad de usar al amigo/a policía para beneficio personal por influencias profesionales y/o facultades que le otorga su trabajo. El objetivo es reírse de la forma instrumental que podría tomar la amistad ahora que nuestro/a amigo es policía. Ocurrido el cambio de bando — se hizo policía— los amigos van a demostrarle discursivamente que no necesitan su nuevo capital, que no les hace falta tener contactos en el otro lado. Le dejarán en claro entonces que su nueva posición no tiene valor positivo en el contexto del grupo de pares amigos. Pero tampoco tendrá valor negativo en este caso. La risa les permite construir una forma de seguir compartiendo, incluyendo al amigo devenido policía a través de la desvalorización 
del poder de policía.

iii) El tercer tipo de chistes se arma sobre el anecdotario vinculado a la labor del amigo/a policía y sus particularidades, como el uso de las armas, ingreso a diferentes lugares, atrapar chorros, pedir documentos. Todas situaciones nuevas en el grupo o realizadas desde nuevas posiciones. Un ejemplo es ponerle nombre al arma o pedírsela para jugar, otra es escucharlo cuando narra acontecimientos sucedidos en el trabajo y recomendarle que actúe de una manera que no está prevista en las situaciones que se enfrenta.

Nuestra interpretación alcanza sobre esta forma de burla dos precisiones. La primera precisión es rescatar la inclusión del anecdotario, ahora policial, en el mismo registro que los anecdotarios de otros miembros del grupo. Lo cual permite hablar entre pares, poner la palabra en común sobre las vidas que cada uno está transitando; ya sea siendo policía o civil, se puede seguir siendo amigo y construyendo un estar juntos. La segunda precisión es cómo esta narración del anecdotario en otro registro, compuesto por contexto de amigos y un tono de comedia - que puede ser colocado por el enunciador o por los amigoshabilita al sujeto a tramitar su doble pertenencia sin escindirse. Como ya indicamos, sin tener que perder su grupo de pares, pero además encontrando un lugar y lenguaje conocidos para compartir una experiencia que pertenece a otro universo de sentido, donde la narración protagónica es la institucional mediada por el lenguaje de informes, sumarios y denuncias. Reírse de los hechos por más trágicos que hayan sido, o justamente por lo dramático, es una traducción de ese lenguaje policial al lenguaje de los pares, y podríamos arriesgar una tramitación saludable del padecimiento subjetivo.

En cualquiera de sus variantes, lo que muestran las jodas (bromas, chistes, cargadas) es la necesidad de poner en relieve la multiplicidad de trazos identitarios que dibujan a estas personas y cómo esto es procesado por el/la policía y sus amigos/as.

El uso de la ironía resuelve simbólicamente las aparentes contradicciones entre ser joven, parte de un grupo de amigos (pensar grupos de jóvenes en el espacio público) y ser policía. Es decir, portar una serie de rasgos y atributos como ser adulto, trabajador, agente del Estado, miembro de una fuerza de seguridad, practicante de la violencia, portador de armas, detener personas, ejercer la ley, representar el orden o ser el que resuelve conflictos y no el que los provoca. Todas éstas, características que entran en tensión con las experimentadas por 
el resto del grupo de amigos.

Esta contradicción — ser joven/policía o ser amigo/policía — llega a fundamentar posiciones de rechazo y expulsión de los grupos, o separaciones, en el caso de algunas parejas. Un ejemplo es un joven militante de un partido de izquierda, quien plantea a sus amigos y compañeros de militancia la necesidad de mejorar sus condiciones laborales entrando a la fuerza policial, pero esto no es comprendido por ellos. A su vez, una de sus amigas es acusada por un ex novio - y ex compañero de carrera universitaria - de formar parte del aparato represivo del Estado, luego de dejar la universidad temporalmente para trabajar como policía.

\section{c) Las parejas}

Los novios y novias también son personas centrales para comprender los alcances e implicancias de ingresar y trabajar de policía. Si bien en algunas situaciones aparecen manifestaciones de apoyo incondicional y comprensión sobre el esfuerzo y las condiciones de trabajo de sus parejas, existen casos donde se da una incompatibilidad explícita como la que describimos en el párrafo anterior.

La comprensión sobre la decisión de ingreso y nuevas condiciones de trabajo, en términos de aceptación y apoyo para con sus parejas se encuentra mediada por la presencia o no de la fuerza en sus propias familias. Pareciera ser que el conocimiento, por parentesco o por afecto, de personas que comparten la profesión que ahora ha elegido la pareja, promueve una actitud de acompañamiento y soporte. Hay un universo de algún modo conocido, compartido a través de la experiencia familiar, que habilita de un modo más fluido la aceptación. Esta circunstancia tiene otra consecuencia favorable para el futuro agente: los familiares políticos serán una red de posibles alianzas y solidaridades para sobrellevar los inconvenientes y sostenerlos afectivamente en el transcurso de la formación y en el posterior desarrollo de la tarea.

Otra situación se produce cuando los nuevos/as policías comienzan a percibir de otra manera actitudes o formas de vida de sus parejas que antes toleraban sin inconvenientes y que ahora, frente a sus nuevas ocupaciones y tiempos, se les tornan difíciles de soportar o por lo menos criticables. El caso más referido son las discusiones por el desequilibrio en el reparto de cargas, horarios, actividades domésticas con las parejas, que serán acusadas de no valorar el riesgo, el esfuerzo que están haciendo al trabajar de policías para el sustento económico familiar. Tener pareja, sostenerla y ser contenido/a afectivamente implica el despliegue 
de una serie de estrategias para todas las personas. Cuando una nueva circunstancia acontece, como es el hecho de ingresar a la policía, el sistema se modifica y hay que restaurar el equilibrio.

¿Y qué ocurre con los que no tienen pareja? En ellos/as la nueva condición influirá en las situaciones de cortejo y expectativa de establecer pareja. Una de las chicas policías dice que en sus salidas nocturnas, al momento de conocer a alguien en un bar o discoteca, su trabajo siempre aparece como tema de conversación:

E: Cuando salís ¿cómo sale el tema cuando hablás con alguien?

Policía: No me creen.

E: ¿Cómo que no te creen?

Policía: No, no me creen, o sea si yo voy a bailar:

-Y... ¿ंvos qué sos?

-Yo soy policía.

—No en serio, ¿vos qué sos?

- Soy policía.

-Eh. Je-je.

Policía: Y como que se van para atrás no sé por qué, je-je. Mis amigos ya saben todos, pero si no el resto no me cree.

$\mathrm{E}:$ ¿Y para conocer gente nueva?

Policía: O no me creen o si no empiezan con el tema de las esposas...

-Y che... ¿ ¿vos usás esposas?

—-Sí, son parte del uniforme.

-Pero... ¿USAS esposas? Ah... y ¿USAS tonzas?

E: ¿Qué cosas?

Policía: Eh... el palo... Como dice Mafalda el palito abolidor de ideologías. Je-je.

Esa es.. y siempre.

— ¿Me prestás tus esposas?

- No no, si querés comprate unas...

Policía: Por ahí los chicos «ganan» más con eso del uniforme, eso «suma» para ellos, han levantado cualquier cantidad de minas. Me ha pasado salir en el móvil con uno y que le tiren besos, le pidan el teléfono, y... ¿ ¿por qué a mí no?

$\mathrm{E}$ : $\mathrm{Y}$ a vos ¿te suma o te resta? Por ejemplo, si viene un pibe y se te acerca en un boliche...

Policía: No, resta.

Decir o callar. Hablar u ocultar su condición laboral es posible en las situaciones donde no deben estar uniformados, pero cuando sí lo están, la visibilidad de su pertenencia institucional tiene consecuencias. 
Por ejemplo, en las prácticas de cortejo heterosexuales la diferencia está generizada. Habrá quienes «sumen» y «ganen» (los varones) y quienes «resten» y «pierdan» (las mujeres). Las posibilidades de encuentro están atravesadas además por la valoración que los otros/as tengan sobre ser policía, y no sólo sobre los estereotipos hegemónicos de masculinidad y femineidad.

\section{TRABAJAR DE POLICÍA: ENTRE LA SEGURIDAD DEL TRABAJO Y LA INSEGURIDAD DE SER POLICÍA}

En la primera mitad del artículo hemos centrado el análisis en las relaciones establecidas por los jóvenes ingresantes con sus familias, novios y amigos. En esta segunda parte, nos ocuparemos del sentido que cobra la seguridad por ingresar al empleo policial, y, al mismo tiempo, las sensaciones de inseguridad, miedo, temor y riesgo que produce dicho trabajo.

Las trayectorias escolares y laborales han sido graficadas por diversos autores como una línea continua entre los estudios primarios, secundarios y superiores, culminando con una certificación de oficio calificado, y un puesto de trabajo. En este esquema, el ingreso al primer empleo posibilitaría hacer carrera, entendiendo esto como crecimiento profesional y salarial de modo sostenido y unidireccional. Estas trayectorias progresivas se presentan acompañadas por una creciente emancipación, que conlleva el abandono del hogar parental para formar su propia unidad familiar autónoma y permanente.

En este camino, estudiar las trayectorias de vida nos permite reconocer lo inadecuado de la interpretación lineal de las trayectorias sociales frente a lo descubierto en esta investigación, porque nuestro caso pone en discusión esas imágenes, pero a su vez las incorpora.

Muchos de los entrevistados ingresan a la policía para resolver una trayectoria laboral que viene a los tumbos; es decir, que está caracterizada por la inestabilidad de empleos de baja calificación, contratos precarios, sueldos bajos, etcétera. Desde ese lugar, la elección de ser policías obedece en parte a la búsqueda de seguridad laboral, es un intento por construir una linealidad que abarca entrar a la escuela de policía, formarse, egresar con un título y un puesto de trabajo del tipo pleno empleo en el Estado.

La seguridad que garantiza este puesto puede leerse como una estrategia de inclusión social, tanto para las personas que ingresan a la policía como para todos aquéllos que consiguen un trabajo de planta 
permanente en el Estado. La pertenencia y estabilidad en el mercado laboral tiene como consecuencia un ascenso en la calidad de vida, tanto en términos individuales como de trayectoria de clase si lo comparamos con los sectores sociales de origen. Es así que el anhelo subjetivo - individual y colectivo-de mejores condiciones de vida, y la situación objetiva de inaccesibilidad en la que muchos se encuentran, o se imaginan a futuro, son circunstancias que empujan la decisión de ingreso a la fuerza. Esta previsibilidad entendida como seguridad en el puesto de trabajo, proyecta en parte una trayectoria laboral lineal; pero como veremos en los próximos párrafos, la tarea de policía incluye condiciones laborales particulares que volverán a situar la no linealidad por medio de la inseguridad y el miedo.

La promesa de un proyecto de vida seguro y estable confronta con la inseguridad y el riesgo que implica el trabajo de policía. La apuesta en juego es muy grande: es la propia vida la que se ofrece a cambio de un salario. La inseguridad significa literalmente la supervivencia donde lo material es el propio cuerpo. El canje entre seguridad laboral e inseguridad que produce el riesgo de perder la vida en el trabajo, aparece en las argumentaciones de los jóvenes policías como una tensión permanente.

\section{a) Los miedos}

Los miedos conforman un conjunto de sensaciones en las que se inscriben y visibilizan las inseguridades vinculada al trabajo de policía. En los discursos de los/as entrevistados/as, estos miedos aparecen en relación a tres grandes temores: i) pérdida de la propia vida; ii) transformación de la personalidad y el carácter; y iii) no poder aguantar esa vida de policías y perder el empleo.

Para comprender el primer temor, es necesario conocer cómo se desarrolla un aspecto de la labor policial y las sensaciones que se le asocian. En ese sentido, identificamos en la descripción que realizan sobre su trabajo un eje narrativo en la oposición inacción-acción que construye temporalidad. Este par configura una experiencia del tiempo de trabajo caracterizada por la dualidad estar al pedo (inacción)/atender un hecho (acción). El primero es un tiempo que, si bien forma parte de la jornada laboral, se lo define como sin acción, que incluye realizar patrullajes y vigilancia de las zonas asignadas a pie, en bicicleta, moto o auto patrullero; de vez en cuando identificar personas o vehículos solicitando documentación; o mantener vigilancia en un objetivo fijo, como 
bancos o instituciones públicas. Es un tiempo en el que no se presentan sobresaltos o encuentros visiblemente violentos. El otro tiempo, de la acción, sucede cuando se dirigen a atender un hecho que implica intervenciones cuerpo a cuerpo que suman un cúmulo de emociones.

La clave de diferenciación de los dos tiempos está constituida por la experiencia emocional que acompañan las acciones; por un lado, el 'embole' o aburrimiento, el transcurrir lento en situaciones sin acción; y por otro, la emoción del vértigo, el miedo, la tensión muscular en las acciones que implican riesgo para sí mismo o para otros. Una de las entrevistadas responde sobre el miedo:

Policía: Y como que al principio no tenés miedo de nada, o sea cuando te mandás, si te dicen están robando en esta casa te metés y no tenés miedo... pero cuando salís te da cagazo que te vayan a dar por la espalda, o sea que te vengan a golpear por la espalda o cuando está la casa oscura y vos no sabés lo que va a haber y te da miedo eso... pero igual la mayoría de los miedos los tenés después, cuando te subís al móvil y te prendes un cigarrillo, o cuando te sentás a tomar mate después de media hora...

—Che... Mirá lo que hubiese pasado si estuviera escondido atrás del sillón.

La descripción anterior nos permite analizar que el temor a la pérdida de la propia vida es a la vez un sentimiento permanente y discontinuo. Es permanente, en tanto es inherente a la labor policial, subyace como si fuera un suelo naturalizado, una sensación latente que no siempre se torna del todo evidente. Pero de tanto en tanto irrumpe el tiempo de acción y el temor en ese momento adquiere un carácter de discontinuo. En ese preciso instante, pero sobre todo luego de la acción — cuando se construye el relato de lo acontecido-, allí es donde el temor vivido se racionaliza, se verbaliza y se asumen los riesgos frente a la posibilidad de perder la vida. Esta experiencia del temor puede suceder en cualquier momento.

El segundo temor, la transformación de la personalidad y el carácter, se experimenta como una emoción duradera, como temor que empieza a instalarse desde que ingresan en la escuela de policía. Es el miedo hacia sí mismos, a cambiar como personas. Es un temor construido a partir de percibir las consecuencias de los cambios de personalidad que se dan al convertirse en agentes de una fuerza de seguridad y cómo esto influye en su subjetividad y relaciones. Es el efecto que se produce en los/as jóvenes aspirantes debido a la formación policial basada en el disciplinamiento de los cuerpos, la asimilación de 
la jerarquía y la obediencia como regla de relación social.

Para el discurso institucional se trata de forjar el carácter. Esta expresión remite a la imagen de un herrero en plena faena: calentar el hierro para moldearlo a golpes y dar una nueva forma que, al enfriarse, será algo permanente. La institución procura producir cuerpos duros, obedientes y disciplinados, que actúen sin vacilaciones ante las urgencias y procesen las órdenes tal cual son requeridas. Este aprendizaje corporal rígido tendrá consecuencias conflictivas cuando se traslade a los otros mundos de significación en que participan los/as jóvenes. Por ejemplo, en la familia, con las parejas y los amigos, los/as jóvenes policías muchas veces reaccionarán de manera diferente frente a las peleas o los requerimientos. Pondrán en acto formas de relación aprendidas en la institución policial que aparecen como disruptivas para el tipo de vínculo que se tiene en los ámbitos familiares, de pareja o amistad. Este carácter duro, intolerante y confrontativo que aparece como novedad en su vida afectiva motiva el miedo a haber cambiado, dejar de ser lo que era y volverse alguien intolerante e intolerable. Un temor a no poder sostener las actitudes y modos de relación con sus vínculos antes del ingreso a la fuerza, que se verán ahora alterados no tan sólo por el cambio de temperamento, sino por no ocupar los mismos lugares, o simplemente no poder dedicarle el mismo tiempo y atención a las actividades compartidas.

El último de los temores es el de no poder aguantar esa vida de policías y perder el empleo. Está vinculado en parte a las condiciones particulares que adoptan las instituciones policiales en Argentina donde los policías suelen verse involucrados, ya sea directa o indirectamente, en las amplias redes de corrupción, clientelismo y/o gerenciamiento de actividades delictivas y el proceder violento que frecuentemente termina en la muerte de otras personas.

Ese contexto laboral implica una toma de posiciones para cada situación, pudiendo involucrarse, hacerse el que no ve o negarse a participar. Cada una de estas opciones llevará a distintas consecuencias, por el enfrentamiento o no que producirá con otros policías o civiles, corriendo el riesgo de perder posiciones en el trabajo, el puesto mismo de trabajo o hasta la propia vida.

En años recientes perder el trabajo como policías se volvió frecuente en la Provincia de Buenos Aires por los cambios realizados desde el Ministerio de Seguridad Provincial. En estos procesos de reforma policial, fueron exonerados (retirados de la fuerza) una gran cantidad de miembros a partir de procedimientos conocidos como purgas Estas me- 
didas instalaron la sensación general de que la continuidad asegurada del puesto de trabajo dejó de serlo, ya que el verse involucrado, directa o indirectamente, en actividades delictivas acarrearía —ahora con más frecuencia que antes - ser expulsado de la institución.

Otra forma de no aguantar es perder la capacidad de sostenerse con el valor, la voluntad y la tolerancia necesarias para trabajar en contextos de uso de violencia. El carácter forjado al calor del rigor y la disciplina en la escuela no garantiza que los agentes no se quiebren y consideren el abandono de la institución como una salida posible.

En todo caso, irse, salir o abandonar, no siempre es una situación que provoque incertidumbre, temor o sea visto como un riesgo. En varios de los entrevistados aparece como un deseo, en tanto se relata el trabajo de policía como un medio para llegar o acceder a otra posición. Por ejemplo, este trabajo estable les permite a varias/os de ellos continuar los estudios terciarios o universitarios. Se registran en las entrevistas expectativas de salir por decisión propia y hacia un trabajo mejor -mejor remunerado, mejor visto y con mejores expectativas personales-, que también se proyecta como distinto a los tipos de trabajo que accedían antes de ser policías. La mayoría de los entrevistados no se proyectaba en el puesto de trabajo policial hasta el retiro. Pensaban este tiempo como un sacrificio temporal, o una circunstancia necesaria, para acceder luego a otro empleo donde encontrarse, o reencontrarse con sí mismos y los suyos de otros modos, teniendo la posibilidad de desarrollar tareas, ocupar el tiempo y habitar sus lugares de pertenencia de maneras diferentes, pero con la experiencia a cuestas de haber sido miembros de fuerzas de seguridad estatales.

\section{CONCLUSIÓN}

En este trabajo nos centramos en el análisis de relatos biográficos de jóvenes que ingresan a las fuerzas de seguridad estatales. Al comienzo del artículo sostuvimos que en Argentina las policías se nutren de mujeres y hombres jóvenes provenientes de clases bajas y medias que finalizaron la educación media. La opción por el trabajo en la policía representa la posibilidad de ascenso en las condiciones materiales de vida frente a trabajos precarios y de baja calificación que a estos sectores les toca. Tomando ese punto de comparación, hemos caracterizado el ingreso a la fuerza como una estrategia de ascenso de clase tanto en términos individuales como colectivos. Ingresar a la policía implica la oportunidad de enderezar trayectorias laborales que hasta 
ese momento, tenían un carácter errante, o que 'vienen a los tumbos'.

Planteamos además que trabajar de o ser policía, es una elección singular, porque el sujeto se incorpora a una institución que ha representado históricamente el vínculo represivo del Estado con sus ciudadanos, y fundamentalmente contra los jóvenes. Además, que el ingreso a la policía produce una serie de cambios en las biografías de los/as jóvenes, no sólo en el plano emocional y afectivo, sino en los modos de narrar la propia historia.

La ruptura, los cambios y/o las transformaciones en la persona y su vida cotidiana adoptan diversas formas en los relatos. En el interés por conocer esos cambios en la subjetividad, y principalmente su impacto en la sociabilidad en los grupos de pertenencia, nos detuvimos en la dimensión afectiva de los/as policías y las sensaciones que experimentan en su formación y trabajo. Llegamos de ese modo a mostrar la dificultad de procesar los cambios que se presentan por parte de su familia, pareja y amigos, o las soluciones simbólicas encontradas por éstos para seguir manteniendo espacios de inclusión, pertenencia y contención. Ejemplo de ello es la utilización de chistes, jodas y gastadas en el grupo de amigos - a través de la ironía y la ridiculización del ser policía - y poder seguir relacionándose como amigos y no como enemigos.

El cambio en la trayectoria laboral y escolar que se produce al ingresar a la institución policial se registra en la narración biográfica marcada por un antes y un después. La vida cotidiana, las sensaciones, el carácter, la valoración de los afectos, de los colores, de la libertad en el uso del tiempo, del cuerpo, de los espacios y la libre elección de la producción de la apariencia, son algunos de las marcas que construyen la distinción entre un antes y un después de estudiar o trabajar en la policía.

Quizás siempre los cambios producen una sensación de incertidumbre y temor, pero en estas biografías hemos podido singularizar la emergencia del miedo en torno a tres cuestiones, que no son lineales, y que permiten complejizar el análisis del binomio seguridad/inseguridad, bajo la paradoja de que al apostar por un puesto de trabajo seguro, aquello los enfrenterá a situaciones de inseguridad de su propia vida.

En síntesis, hemos intentado demostrar que las trayectorias lineales no siempre deben ser leídas como trayectorias seguras. El oficio policial es una profesión de larga data, probablemente no haya novedad en varios aspectos de la condiciones laborales que rodean la tarea en la fuerza. Hace también mucho tiempo que son los/as jóvenes esco- 
larmente exitosos — que terminan el nivel de educación media- provenientes de sectores bajos y medios-bajos los que ingresan a la fuerza. Y como bien rememoramos, es también antiquísimo el vínculo conflictivo juventud/policías. En el contexto de una realidad siempre cambiante, resultó interesante investigar la forma que toman las biografías de jóvenes policías, y cómo resolvieron sus trayectorias escolares y laborales, aportando también a la comprensión de las lógicas simbólicas que cohesionan las pertenencias identitarias y en un mismo movimiento nominan el nosotros/amigos y los otros/enemigos.

LA Plata (ARGENTINA), ENERO 2011

RECIBIDO: FEBRERO 2011. ACEPTADO: MARZO 2011.

\section{REFERENCIAS BIBLIOGRÁFICAS}

BOVER, TOMÁs (2010): «Trabajar de policía: entre la seguridad del trabajo y la inseguridad de ser policía». Actas de la II Reunión Nacional de Investigadoras/es en Juventudes Argentina. Salta, Universidad Nacional de Salta.

— y S. CALANDRÓN (2009): «De-formar a la fuerza. Un análisis de las reformas policiales en los espacios de formación profesional». Actas de la III Reunión de Antropología del Mercosur. San Martín: Universidad Nacional de San Martín.

CHAVES, MARIANA (2010): Jóvenes, territorios y complicidades. Una antropología de la juventud urbana. Buenos Aires: Espacio Editorial.

_ (2009): «Investigaciones sobre juventudes en Argentina: estado del arte en ciencias sociales 1983-2006». Papeles de Trabajo No5. Buenos Aires: IDAES. Disponible en: www.idaes.edu.ar/papelesdetrabajo.

— (2007): «Procesos de formación policial en la Provincia de Buenos Aires». Actas de las Séptimas Jornadas de Sociología. Buenos Aires: Universidad de Buenos Aires.

— (2005): «Juventud negada y negativizada: representaciones y formaciones discursivas vigentes en la Argentina contemporánea». Última Década $\mathrm{N}^{\circ} 23$. Valparaíso: Ediciones CIDPA.

FREDERIC, SABINA (2008a): «No me quiero morir en esto: usos del tiempo y configuración del oficio policial en el Gran Buenos Aires». Actas de las V Jornadas de Sociología. La Plata: UNLP.

(2008b): «Los usos de la fuerza pública: debates sobre militares y policías en las ciencias sociales de la democracia». Universidad Nacional de 
General Sarmiento Los Polvorines. Buenos Aires: Biblioteca Nacional. Sirimarco, Mariana (2009): De civil a policía. Buenos Aires: Editorial Teseo.

(2004): «Acerca de qué significa ser policía». SOFí́A TisCORNIA (compiladora): Burocracias y violencia: estudios de antropología jurídica. Buenos Aires: Editorial Antropofagia. 